\title{
Saber, manipulación y poder. La universidad como institución social ${ }^{*}$
}

\author{
Napoleón Murcia Peña ${ }^{* *}$, Audin Aloiso Gamboa Suárez ${ }^{* * *}$
}

\begin{abstract}
RESUMEN
Recibido: 2 de julio de 2014

El texto es una reflexión sobre la universidad como institución

Evaluado: 6 de agosto de 2014 social, realizada a partir de varios estudios desarrollados en los Aceptado: 26 de agosto de 2014 cuales se evidencia el interés de algunas estancias por convertirla en un escenario de manipulación y control, lo que la reduce a la racionalidad de los organismos funcionales, frente a la dinámica propia que la universidad instaura como institución social, cuya base se moviliza desde las significaciones imaginarias sociales. En este proceso, se muestra desde la lógica de la teoría de los imaginarios sociales la forma como la universidad se configura e institucionaliza en una tensión constante, entre el saber y el control, entre la función y la institución, y se dan las bases que la definen y posicionan como institución imaginaria social.
\end{abstract}

Palabras clave: universidad, saber, poder, imaginarios sociales.

\footnotetext{
Los estudios en los cuales se basa esta reflexión son "Sistema de autoevaluación universitaria con miras a la acreditación de alta calidad" (Murcia, Sánchez y Candamil, 2006); "Vida universitaria: un estudio desde los imaginarios sociales de profesores y estudiantes" (Murcia, 2006, 2012); "La educación ambiental en la Universidad de Caldas" (Ospina y Murcia, 2007, 2008). Cómo citar este artículo: Murcia Peña, N. y Gamboa Suárez, A. (2014). Saber, manipulación y poder. La universidad como institución social. Hallazgos, 11 (22), 419-434.

* Doctor en Ciencias Sociales Niñez y Juventud de la Universidad de Manizales (CINDE), posdoctorado en Narrativa y Ciencia de la Universidad Santo Tomás y Universidad de la Plata (Argentina) y magíster en Educación de la Universidad de Caldas. Docente investigador de la Universidad de Caldas, Colombia. E-mail: napo2308@gmail.com

${ }^{* * * *}$ Magíster en Pedagogía de la Universidad Industrial de Santander y candidato a doctor en Ciencias de la Educación (Rudecolombia). Profesor Investigador de la Universidad Francisco de Paula Santander. Cúcuta, Colombia. E-mail: docaudin@gmail.com
} 


\title{
Know, Handling and Power. The University as a Social Institution
}

\begin{abstract}
The text is a reflection on the university as a social institution, made from several studies whose findings are developed in evidence, first, the interest of some romos to turn it into a stage of manipulation and control, reducing it to the rationality of functional agencies, compared to the dynamics that the university established as a social institution whose base is mobilized from the social imaginary significations. In this process, is shown from the logic of the theory of social imaginary the way the university is set and institutionalized in a constant tension between knowledge and control, between the function and the institution and the foundation are given the defined and positioned as imaginary social institution.
\end{abstract}

Keywords: University, namely, power, social imaginary. 


\section{Saber, manipulação e poder.}

\section{A universidade como uma instituição social}

\section{Resumo}

Este texto é uma reflexão sobre a universidade como uma instituição social, realizada a partir de vários estudos desenvolvidos nos quais é evidenciado o interesse de algumas entidades por convertê-la num cenário de manipulação e controle, o que tem-la reduzido à racionalidade dos órgãos funcionais, frente à dinâmica própria que a universidade propõe como instituição social, na qual sua base mobiliza-se desde as significações sociais. Neste processo, é mostrada desde a lógica da teoria dos imaginários sociais a maneira como a universidade configura e institucionaliza em uma constante tensão, entre o saber e o controle, entre a função e a instituição, e se dão as bases definem-lha e posicionam como instituição imaginária social.

Palavras-chave: universidade, saber, poder, imaginários sociais. 


\section{INTRODUCCIÓN}

La Universidad, concepto nacido en el medioevo, significó la unidad de cosas diversas o unidad en la diversidad y, en el mismo sentido, unidad de personas congregadas en un gremio social denominado como corpus, collegium, communio, societas o consortium, y referido exclusivamente al grupo dedicado al saber. Relata Borrero (2005) que a finales del siglo XII aún se hablaba de la congregación de los maestros de Paris o consortium magistro rum parisientium. Este carácter de agremiación asociativa es la que le ha permitido mantenerse, a través de la historia, como institución social y ejercer autónomamente las funciones que le son inherentes, devenidas de lo que originariamente se denominó como sus notas sustantivas de entidad corporativa, universal y científica, a lo que se le sumaría su carácter autónomo.

Dos condicionantes surgen en esta consideración: una referida al estatus otorgado a la universidad como centro del saber y la otra referida a la forma organizativa que toma la universidad desde sus orígenes.

El origen mismo de la universidad se asienta en imaginarios de poder, de dominio del conocimiento para ejercer ciertas influencias sobre las diversas esferas de la vida humana. El poder del pensamiento para liberar al ser humano del dominio natural es una idea que se venía configurando para darle forma y piso científico y teórico a unos mecanismos de manipulación inicialmente religiosos y ortodoxos, que consideraban el ascetismo como el mejor medio para lograr esa personalidad sumisa y obediente de las leyes divinas; y posteriormente positivista y conductual, que buscó la emancipación de estos supuestos desde la racionalidad fría e instrumental y la dominación del hombre y la mujer mediante la imposición de otra realidad única e inmutable: la realidad del cientifismo positivista. Esta postura es compartida por historiadores como Zarruk (1992), considerando incluso como una potente influencia en el origen del nuevo poder de la Edad Media: el poder del saber.

Tal vez por eso, para facilitar esta expresión social de dominio religioso, buscaban reunir estudiantes de todas los países en una especie de "Universitas Stadiorum", que formaban las grandes élites de futuros gobernantes y propietarios del poder, lo que daba origen a los colegios universitarios, creados como hospederías y convertidos posteriormente en centros de educación o Facultades, como el caso de la Universidad de Salamanca o las universidades de Oxford y Cambridge (Romero, 2006).

Pese a que representaron la cúspide de la sabiduría de la Edad Media, en el Renacimiento comenzaron a declinar por atenerse a sus tradiciones escolásticas y no admitir, más que tardíamente, las nuevas ciencias, justamente por la fuerza de las estructuras imaginarias que se imponían en la época. Esto debido a la resistencia al cambio que generan los imaginarios fuertemente instituidos, prevista por Shotter (1993).

Pero las condiciones sociales estaban cambiando, la dependencia de lo mítico y religioso estaba encontrando duras críticas, y los imaginarios sociales apuntaban a otras esferas más productivas. La formación pasaba a manos de laicos, quienes se oponían a los procesos dogmáticos de formación religiosa, creando, a finales de la Edad Media, 
las escuelas gremiales, lo que dio origen a una orientación eminentemente profesional de la universidad. En este marco de tecnificación para la producción, en las ciudades se crearon escuelas municipales, independientes de las claustrales y catedrales, cuyo carácter era esencialmente práctico; sin embargo, en algunas se enseñaban asignaturas de carácter humanístico como Literatura, Geografía e Historia.

Los imaginarios de las sociedades del renacimiento del siglo XV eran bien diferentes, dando origen a la educación humanista, considerada por muchos como la educación moderna. Las estructuras de los imaginarios se orientaban a resaltar otro tipo de poder, el poder del hombre como amo y dueño del mundo. En torno a esta nueva lógica se mueven las universidades del Renacimiento, y surge así una cantidad de maestros que comienzan a pensar y escribir sobre educación, influidos por la educación científica desde las propuestas renovadas de Galileo y Keplero.

Sin embargo, en la España colonial, de donde deviene el origen de nuestras universidades, el poder del clero era evidente y con ello las oposiciones rotundas a las transformaciones, tanto teóricas como organizativas de la universidad. Esta influencia se ejercía sobre todas las cosas de universidad, a tal punto que para funcionar debería tener el reconocimiento de los más altos prelados de la Iglesia otorgado mediante la bula papal. Aquí el imaginario sobre universidad estaba inscrito en la idea de control y poder religioso, desde cuyos principios se crearon las primeras universidades en América (México, Lima, Chile, Caracas y Guadalajara). En Colombia en 1958, se creó la Universidad de
Santo Tomás, en Bogotá, bajo la dirección de la comunidad de Santo Domingo, en la cual trabajaban arte y teología. Posteriormente, la comunidad de San Ignacio de Loyola fundó el Colegio Máximo, donde los contenidos se orientaban a la Ration Estudiorum de la orden jesuita. De hecho, todas las universidades deberían funcionar con la Ration Estudiorum de su comunidad fundadora y seguir los contenidos del trivium y quadrivium (Borrero, 2002).

Aunque el control de la universidad en América era total por parte de la Iglesia, a finales del siglo XVII y comienzos del XVIII aparecen algunas influencias del movimiento cultural de la ilustración que comienzan a generar transformaciones en estos imaginarios, a tal punto que llevaron a reformar los planes de estudio introduciendo las teorías de científicos que contradecían los postulados religiosos. Como el caso de la Universidad Javeriana, que comienza a desarrollar en sus planes de estudio las teorías de Newton y Copérnico.

En este ambiente de influencias europeas, se produce entonces la expulsión de los jesuitas de la Nueva Granada y se comienzan a desarrollar propuestas autónomas de la élite criolla sobre la universidad pública, en las cuales se reformaba el método escolástico por el experimental. Sería el general Moreno y Escandón (1736-1792) quien presentó la primera propuesta para la universidad pública, en la cual, por primera vez, el Estado tendría control sobre ella, dando relevancia a los contenidos aplicables y prácticos. Para Moreno y Escandón (1774, citado por Arango, 2005) los religiosos tenían una obsesiva enemistad hacia los avances de la ciencia y la filosofía útil. 
Pero con esto, el imaginario de universidad como poder y dominio solo tiene cambio de escenario, pues en la naciente Nueva Granada se comienza una gran pugna por el control de la universidad, la cual se canaliza en dos direcciones: la primera, en el marco del poder explicativo y predictivo de la ciencia que se debería desarrollar en la universidad, en contraposición al poder religioso del trívium y quadrívium; y la segunda, enmarcada en la pugna por su dominio administrativo, expresada entre quienes consideraban que debería estar bajo las orientaciones del Estado, independientes del poder eclesial y quienes asumían la necesidad del domino de las ideas religiosas y de la herencia de la Corona española.

Esta pugna es reflejada en el traslado que Gutiérrez de Piñérez le hiciera a Moreno y Escandón para lima, buscando opacar con ello las nuevas ideas del general. Traslado que facilitó la contrarreforma llevada a cabo en 1778 y que devolvió el poder al clero. Una serie de eventos sucedieron, algunos buscando mostrar la independencia de la universidad pública como la expedición de Mutis y otras, lo que demostró el dominio de las tradiciones escolásticas como las persecuciones posteriores impulsadas por el virrey José de Ezpeleta (1789-1796) contra quienes divulgaran los ideales de la revolución francesa en la universidad.

Pese a ello, los ideales de la Ilustración se irían reconociendo progresivamente en la educación universitaria hasta influir en decisiones posteriores protagonizadas desde los partidos políticos de la nueva granada por Bolívar (1783-1830) y Santander (1792-1840), el primero, representado los intereses religiosos del conservatismo y el segundo, los del liberalismo transformacionista de la época. Pugnas que se evidencian en las constantes reformas dadas en el siglo XIX, algunas centradas en los ideales reformistas de Bentham y otras que impulsaban las ideas religiosas de Santo Tomás de Aquino (Manchola, 1993 y Luzurriaga,1976).

\section{INSTITUCIONALIZACIÓN DE LA UNIVERSIDAD: ORGANIZACIÓN MECÁNICO FUNCIONAL VERSUS INSTITUCIÓN SOCIAL}

Los estudios tomados como referencia dejan entrever la fuerza de la tradición instituida en la universidad, que la asume como una institución meramente económico-funcional, en la cual no solamente se asume una estructura estable de ella, sino que se valora desde las lógicas de la producción.

Esta perspectiva tiene relación con los imaginarios de orden, mercado y capital que se han instaurado en la universidad desde los cuales se definen sus políticas. Considerar la universidad como institución "económico funcional" (Castoriadis, 1983) significa predefinirla desde prospectivas que se formulan en logros y metas ideales y fijas, estructuradas fundamentalmente, en términos de cobertura, repitencia, sostenimiento y producción; categorías analíticas desde las cuales se mide el impacto de la educación con miras a la eficiencia y eficacia. Los procesos de autoevaluación en proyección a la "acreditación institucional de alta calidad", propuestos por el Consejo Nacional de Acreditación (CNA), son la síntesis de la visión funcional de la universidad ${ }^{1}$. Este análisis coincide con

1 Las categorías propuestas por el CNA asumen métodos de inducción analítica, reduciendo la autoevaluación a la descripción y tabulación 
el realizado por Porter (2005) desde su estudio de la universidad mexicana:

\section{[...] la universidad de papel, en sus pá- ginas se va dibujando esa institución imaginaria en la que se impone la su- premacía del procedimiento como jui- cio sumario de comportamientos que, al ser codificados mediante datos, han quedado por ello mismo vaciados de contenido [...] (p. 8).}

Esta racionalidad propia de un imaginario de mercado envuelve los indicadores que la universidad colombiana exhibe como sus logros. De hecho, tanto la propuesta de acreditación del CNA, como los procesos de postacreditación, definidos en los planes de desarrollo y sus programas, se fundan en esas racionalidades que Castoriadis (2008) ha denominado "conjuntistas", en cuanto fundadas en la racionalidad instrumental de las agrupaciones fraccionales de conjuntos y la expansión del dominio racional, la cual se encarna en la cuantificación como criterio de autofundación. Esta tendencia se ha naturalizado en el tratamiento de la universidad y ha llevado a la búsqueda de resultados desde fundamentos y certidumbres absolutas que se definen desde la supuesta necesidad de universalidad del conocimiento.

numérica de escenarios y procesos (basta con observar en los factores propuestos, las categorías definidas para el análisis las cuales se centran en aspectos meramente funcionales). Por ejemplo, en lo académico se proponen categorías como la interdisciplinariedad, flexibilidad, evaluación del currículo y programas, pero el interés es meramente técnico, por cuanto propone en las estadísticas la búsqueda de los factores causales de problemas sin trascender hacia la emergencia de categorías propias del entorno y la comprensión de lo que en realidad pasa en la universidad cuando se apoya uno u otro enfoque académico. El problema en la universidad no necesariamente se encuentra en la falta de flexibilidad, o de interdisciplinariedad, puede estar en otro factor mucho más profundo que es el modelo de la universidad colombiana.
Los planes de desarrollo y mejoramiento, y sus proyectos derivados están siempre definidos desde sociedades ideales, cuyos límites son las cantidades: cantidad de magíster o doctores, cantidad de productos por maestro, cantidad de estudiantes por espacio, cantidad de textos en las bibliotecas, cantidad de estudiantes promovidos. Los espacios dados en los instrumentos de control y evaluación no contemplan en absoluto el espacio para la comprensión de las circunstancias que hacen posible la aparición de un fenómeno social. Los pocos espacios son ampliación de las afirmaciones, preestablecidas por el índice o indicador definido de antemano por la universidad.

Pero también lo son los sistemas de control interno de las universidades. Las evaluaciones académicas están definidas por representaciones simbólicas que agrupan los buenos y malos estudiantes, los sistemas de evaluación docente califican el desempeño en condición de castigar y sancionar las formas de valorar las producciones de los maestros que hacen desde la cantidad de sus productos. Esta tendencia se radicaliza en la actualidad con la racionalidad totalmente conjuntista desde la cual se miden los grupos de investigación e investigadores en Colciencias, razón a la cual se apegan cada día más las universidades en Colombia para realizar sus exigencias a los maestros.

Sin embargo, a pesar de estas prácticas instituidas desde el mercado y consumo, y pese a esa fuerza de lo instituido que hace corriente el considerar que la universidad como organización económico/funcional, cuando se estudian los imaginarios sociales desde su historicidad, se muestra que su verdadera naturaleza es su institucionalización 
dinámica siempre configurada por ese "colectivo anónimo" que la configura y se configura con su creación.

Los estudios de referencia muestran que la universidad es, ante todo, una institución social compleja y por tanto constituida por la sociedad, pero a la vez constituyente de sociedad y universidad (Murcia, 2006; Murcia, Sánchez y Candamil, 2006; Murcia Peña, Murcia Gómez y Murcia Gómez, 2010; Murcia, 2012). Es, a la vez, generada y generante, dinamizada y dinamizante. Es una institución social que se instaura en un magma de significaciones imaginarias sociales y a la vez influye sobre ese magma y lo reconstituye, porque en la universidad se funden aspectos sicosomáticos, sociales, culturales, racionales e históricos para definir una condición de acuerdo social, desde el cual las personas que viven en ella organizan sus vidas, reconstituyéndola constantemente.

La fundación, por ejemplo de la Universidad de Caldas, correspondió a una serie de intereses sociales que en el momento de su configuración imperaban en la región; en ella se plasmaron también los ideales de esa casta de campesinos y ganaderos que pensaron la Facultad de Agronomía y Medicina Veterinaria y se consolidó la idea de los jóvenes artistas con la Facultad de Bellas Artes. La forma como se organizó la universidad también correspondió al desarrollo histórico social de la época, por eso, siguiendo las recomendaciones dadas desde el movimiento estudiantil de 1919 en Córdoba, se nombró una junta directiva que involucrara representantes de estudiantes y maestros (Murcia, 2006).

Efectivamente, el colectivo anónimo, como define Castoriadis (2008), es la fuerza social que crea la institución social desde sus significaciones imaginarias y desde estas significaciones genera unas funciones que ayudan a que la institución creada pueda ser posible, pero al crear la institución se establece también una nueva sociedad desde lo creado por ella.

En este marco, el padre Alfonso Borrero muestra cómo la universidad desde su fundación en el siglo XII ha obedecido a un proceso de construcción histórico social devenida de las más enconadas dinámicas de transformación y cambio, pero también impulsora de esos cambios y dinámicas. En esta medida, presenta a la universidad como escenario y origen de saber y por tanto como escenario y origen de autonomía y construcción social, donde los imaginarios respecto de las sociedades y las ciencias han llevado a forjarla como institución autónoma. Pese a su naturaleza autónoma y social de origen, históricamente se han generado también intentos de manipulación y juego de poder, como ya se dijo. En síntesis, Borrero (2005) en su obra deja ver un haz de incertidumbre y sospecha sobre las "estructuras" aparentemente instituidas de la universidad, que la han hecho objeto de manipulaciones y estancos, y la reflexión sobre nuestra posibilidad y responsabilidad de continuar construyéndola como entidad autónoma y libre.

La misma dinámica social hace que los sujetos tomen lo que ya está y lo carguen de sentido privado, constituyan "cada vez su orden simbólico [...] Pero esta constitución no es 'libre', debe también tomar su materia en 'lo que ya se encuentra ahí'" (Castoriadis, 1989, p. 208). De ahí que sea peligroso que las instituciones, pese a ser creadas por lo social, olviden su trayecto de creación social. 
Un funcionalista puede considerar como evidente que, cuando una sociedad se otorga a sí misma una institución, se da al mismo tiempo como posibles todas las relaciones simbólicas y racionales que esta institución conlleva o engendra-o que en todo caso no podría haber contradicción o incoherencia entre los fines funcionales de la institución y los efectos de su funcionamiento real y que cada vez que se plantea una regla, queda garantizada la coherencia de cada una de sus innumerables consecuencias con el conjunto de las demás reglas ya existentes y con los fines consientes $\mathrm{u}$ objetivamente perseguidos (Castoriadis, 1989, p. 211).

De ahí que las dinámicas que muestra la universidad no la encierran en esta consideración (económico/funcional); por el contrario, lo que se evidencia es la ebullición siempre inconclusa y efervescente de la institución que busca en cada momento formas diversas de proyectarse y de subsistir como institución en la medida de las trasformaciones de las significaciones imaginarias sociales.

\section{LA UNIVERSIDAD COMO}

\section{INSTITUCIÓN IMAGINARIA}

La sociedad es una institución constituida por la misma sociedad e influida por su propia constitución. Es decir, antes que todo, la sociedad es construcción social y sus simbólicos e imaginarios solo tienen sentido en el marco de esas estructuras o esquemas de significatividad que la definen y que ella misma ha definido (Murcia, 2012).

Por supuesto, implica que es la sociedad la que crea por sí y para sí un magma de significaciones desde el cual organiza su propio mundo, pero a la vez es organizada por ese magma creado. En ese magma hay ebullición de representaciones particulares y colectivas desde múltiples influencias: naturales, biológicas, sociales, individuales, que al fundirse constituyen las pautas y herramientas para organizar el mundo de esa sociedad determinada, pero que a la vez son constituidas por esa sociedad.

Castoriadis (1989), diría que

Esta institución es en cada momento
institución del mundo, como mundo
de esta sociedad y para esta sociedad,
y como organización-articulación de
la sociedad misma. Suministra el con-
tenido, la organización y la orienta-
ción del hacer y del representar/decir
sociales. Lleva inexorablemente con-
sigo, como creación de la sociedad, la
institución del individuo social, por
medio de ese teukhein y del hacer
particular representados por la socia-
lización de la psique /soma. Por ello,
la sociedad da existencia a los indivi-
duos (p. 329).

En otras palabras, para instituir la universidad, la sociedad crea las bases funcionales desde las cuales las personas pueden hacer cosas, representarlas o decirlas sobre y en la universidad, teniendo la seguridad de que si están dentro de esos acuerdos creados, dentro de esos límites establecidos, será factible su inteligibilidad social, pero sobre todo lo que se diga, haga o represente en ese marco funcional validado podrá ser creíble, confiable y reconocido como realización, discurso o representación "de la universidad " (plausibilidad social). 
De este modo la sociedad crea las bases funcionales de la institución social, lo que Castoriadis (1989) llama la institución del legein y teukhein social, que implica crear los acuerdos sobre las formas de ser/hacer, decir y representar de las instituciones sociales. Estas bases funcionales descansan sobre las significaciones imaginarias sociales $\mathrm{y}$, por tanto, son ellas las que las definen, dinamizan y transforman.

Esta particularidad hace que las instituciones sociales no sean meras organizaciones funcionales, pues al reconocer las funciones como devenidas de las significaciones imaginarias sociales es en ellas donde debe centrarse cualquier mirada para viabilizarla y transformarla. De esta forma, las bases funcionales creadas se constituyen en puntos de referencia que se movilizan desde la misma dinámica de los imaginarios sociales que la definieron como posibilidad. A propósito, Baeza (2000) considera que

La institución es el conjunto de significaciones legitimadas de manera social, independiente de una funcionalidad precisa; la institución remite por tanto al ámbito de las aceptaciones colectivas, de las ideas, de las fantasmagorías, etc. que pasan a formar parte de nuestro sentido común. La sociedad es justamente una institución mayor (p. 26).

Antes que todo, la institución es movilidad de significaciones comunes y no comunes; por tanto, la creación de la universidad no obedece a un proceso de yuxtaposición de situaciones y funciones que fungen como normatividades inalienables por cumplir. No es la definición de una institución ideal desde la imposición de sus normas y leyes que harán que la sociedad o institución funcione armónicamente desde las direcciones preestablecidas (como lo pretenden mostrar los imaginarios hegemónicos, que buscan la reproducción de las "estructuras sociales").

Por el contrario, la creación de la universidad obedece a un proceso caótico; en ocasiones desenfrenado y sísmico, donde se conjugaron componentes fundidos de toda una historia social, política, económica, cultural, pero también psicosomática. No son las funciones las que definieron la universidad, sino las convicciones y creencias/fuerza sobre la naturaleza del mundo, del ser humano y de su deber ser. No fue el imaginario aislado de una persona o una fuerza extraña por fuera de las personas lo que dio origen a la universidad, sino fuerzas psicosomáticas y sociales que articularon esas convicciones/creencias fuerza surgidas de los intereses y necesidades, en acuerdos sociales sobre los formas de ser/hacer, decir/ representar esta institución, pero que a la vez que se iban logrando, que fueron influyendo las mismas fuerzas, pues se instauraron como nuevas creencias, convicciones/ fuerza social. Como lo afirma Schutz (2008), fuerzas que se estructuraron como "ámbitos finitos de sentido", con unos propósitos e intencionalidades concretas, empujadas por las significaciones imaginarias sociales.

En este sentido, los cierres o limites que la sociedad traza a la universidad mediante la delimitación funcional del teukhein y legein no corresponden a algo dado, establecido de por sí; por el contrario, son los actores quienes colocan esos cierres de acuerdo con los límites mismos de nuestras conciencias. Por eso, las instituciones sociales son, en la medida de la capacidad del ser humano, 
como lo dijera Foucault. De ahí que dependa de los actores sociales el hacer de estos esquemas inamovibles y estáticos, o asumir esas fronteras como relativas y dinámicas. Shotter (1993) lo plantea en términos de que incorporamos desde las conversaciones cotidianas cierres sobre las instituciones, las cuales van logrando un estatus tal que se constituyen en instituidas, y mientras más se hable de estos más se instituyen socialmente. Según el autor, cuando estas referencias son cooptadas por el Estado se vuelven estáticas y pierden su naturaleza imaginaria. He ahí la estructuralización de la universidad.

Pero además, la sociedad es magma de magmas, porque en ella se conjugan todas las funciones del mundo: lo histórico, social, individual, natural, racional, imaginario. Por eso, cuando se crea la universidad, lo que hacemos es delimitar de ese gran magma un fragmento y lo dotamos de identidad. Una identidad que es construida por la sociedad desde sus significaciones imaginarias y que al tiempo la influye; un fragmento en el que están contenidos todos los componentes fundidos en el gran magma social.

Toda institución social es validada y reconocida por quienes está instituyendo, mediante los dispositivos simbólicos generados en el marco de unas significaciones imaginarias sociales, que, a la vez que constituyen la institución, se dejan constituir por sus actores, a tal punto que los dispositivos simbólicos deben ser la forma de orientación y expresión de un magma de significaciones sociales que no solo orientan a las personas, sino que les permite reconfigurar constantemente dichos simbólicos. Castoriadis (1983) lo precisa cuando expone que la institución
[...] consiste en ligar a símbolos (a significantes) unos significados (representaciones, órdenes, conminaciones o incitaciones a hacer o a no hacer, unas consecuencias- unas significaciones, en el sentido lato del término) y en hacer valer como tales, es decir, en hacer valer este vínculo más o menos forzado para la sociedad o el grupo considerado (p. 201).

De hecho, la universidad existe porque la sociedad ha sido capaz de fundir símbolos y significados sociales en unos acuerdos que nunca dejan de ser temporales, por eso existe el Proyecto Educativo Institucional (PEI) y todas las normatividades en la universidad.

Así, la educación-formación, objeto de la universidad, es uno de los constructos simbólicos más ricos y complejos que la sociedad ha imaginado, para introducir a los individuos a ese magma de significaciones expresadas en el gran mundo simbólico de lo cultural, comunicativo, político, ético y estético $\mathrm{y}$, en tal razón, debe propiciar su movilidad. Como extracto de ese gran magma social, debe también considerarse su dinámica constante en dirección a autoconstituirse en medio de las influyentes polifonías de quienes afecta, más no así solamente reproducirse, como lo definirían las propuestas estructuralistas.

\section{LA UNIVERSIDAD COMO INSTITUCIÓN SOCIAL}

La universidad es un escenario social, cultural, político, ético-estético y cognitivo donde se confrontan constantemente ideas, sentimientos y proyectos, pero sobre todo donde se vive 
y se comparten experiencias, teorías y sensibilidades que pretenden ayudar a mantener, construir y desarrollar al individuo, la sociedad y la cultura (Murcia, 2012, p. 36).

La universidad como institución social no es más que la organización de un conjunto de acuerdos sobre la educación/formación en relación con sus más altos estándares, legitimados por la sociedad, en cuyos linderos se juega la posibilidad de hacer comprensibles y válidas las acciones e interacciones de sus actores. Por ser el producto de la conciliación de significaciones imaginarias sociales en simbólicos, que definen las formas de ser corrientes en ella, la comunidad es actora real de cada acontecimiento que en la institución se genere. El rol de sus miembros cambia por el de actores sociales, quienes antes que "cumplir con una función asignada" buscan dar algo de sí en cada acción o interacción; buscan aportar de su propia cosecha algo que ayude a consolidarla en los más altos niveles de calidad; buscan agregar un valor a lo ya definido, matizando los acuerdos (proyectos y leyes) con sus propias voces, en la medida que logran hacer suya la universidad.

Gracias a que es instituida desde y en un magma de significaciones imaginarias sociales, está sujeta a la implacable ebullición y fusión entre lo sicosomático, lo histórico, lo cultural, lo racional y lo imaginario. Por tanto, al desequilibrio y equilibrio constante entre lo instituido, instituyente y radical. Se evidencia en las diferentes luchas de fuerzas, las que quieren mantener unos criterios de universalidad y las sociales instituyentes y radicales que buscan suportar otras alternativas.
Es acuerdo y posibilidad, en consideración a lo cual es definición y redefinición de funciones y estatus de cada una de ellas y es creación de condiciones para que esas funciones sean posibles. Pero sobre todo es creación y autopoiesis, no como sistema, pero sí como institución magmática que se genera y regenera constantemente en el marco de ebulliciones confusas y caóticas que, a veces siguen la autorreferencia propia de la autopoiesis, pero que a veces se sale de ella para generar nuevas formas de organización, que con el paso de la presión instituyente se van consolidando en imaginarios instituidos incluso por el Estado.

Por esto, ese límite establecido por lo social en el teukhein y legein ${ }^{2}$, es tan solo referencia para las acciones e interacciones de sus actores, pues en medio de la ebullición que implica su funcionamiento, ellos construyen un mundo especial donde se funde lo instituido, pero también lo radical y lo instituyente. Un mundo que se desplaza en los bordes de lo que el estado ha adoptado como propio, o que la universidad, mediante sus reglamentos y planes, ha concebido como su ordenamiento. Esta característica está en concordancia con lo expresado por Castoriadis (1983), cuando afirma que “Ninguna sociedad puede existir si no organiza la producción de su vida material y su reproducción en tanto que sociedad. Pero ninguna de estas organizaciones son ni pueden ser dictadas indefectiblemente por unas leyes naturales o por consideraciones racionales" (p. 252).

Pese a que se han construido y acordado los trazos del sendero que la universidad debe

2 Categorías de los acuerdos sociales desde las significaciones imaginarias sociales, referentes al hacer/decir y representar. 
recorrer, los actores transitan por los bordes de sus límites, definiendo sus propios recorridos, retando los altibajos del relieve $\mathrm{y}$ reconstruyendo sobre lo trazado nuevos trazos, e incluso definiendo nuevos senderos y puntos de llegada. Las leyes, normas, reglamentos y planes - así hayan sido construidas con la participación social - nunca permanecen estáticas en estructuras inamovibles, pues estos límites en la vida social de la universidad no son nítidos, y los actores salen y entran en ellos constantemente, desplazando incluso sus fronteras. De ahí que estas sean sólo una guía importante en el hacer, representar e imaginar la universidad. Por ejemplo, siendo que su naturaleza es pública, nadie podría adoptar comportamientos, discursos o representaciones propios de una universidad privada, los hallazgos en los procesos de investigación anunciados como referente son contundentes:

En realidad, el mundo social de la universidad no se construye en esos simbólicos instituidos (oficiales o institucionales), pues ellos son apenas formas ideales que han sido plasmadas o no, con amplios procesos de participación social; es claro que en la medida que ese instituido responda a las exigencias de inteligibilidad social, en la medida que sea sancionado por la comunidad, en esa medida es seguido por ella; pese a ello, es claro también que no es en ellos, ni desde ellos, exclusivamente que se construyen la vida social de la universidad, sino que esta se desarrolla en los bordes de esto instituido oficial o institucionalmente, en los bordes de eso que ha sido racionalizado, pensado y organizado (Murcia, 2006, p. 256).
Como se ha argumentado, la institucionalización de la universidad siempre ha sido un proceso constructivo del colectivo anónimo, que atiende a esa gran base de significaciones imaginarias sociales, pero no por ser acuerdo o imposición, la universidad se estatiza en sus esquemas creados. Lo que se evidencia en los procesos de investigación es que, al contrario, cuando se hace el seguimiento histórico de las categorías, estas se van trasformando en la medida de las trasformaciones de esas significaciones imaginarias sociales.

Los desequilibrios comienzan por las salidas de las comunidades hacia los bordes de lo establecido como normal. Estos imaginarios radical/instituyentes van generando sus propias representaciones simbólicas hasta configurarse en imaginarios fuertemente instituidos o bien por la práctica social o por coacción institucional. Con razón, Castoriadis (1989) afirmaría que "Realidad, lenguaje valores, necesidades, trabajo de cada sociedad, especifican en cada momento, en su ser particular, la organización del mundo y del mundo social referida a las significaciones imaginarias sociales instituidas por la sociedad en cuestión” (p. 330).

Dichas realidades se reconfiguran constantemente, en la dinámica misma de las reconfiguraciones de los imaginarios sociales. Así, las instituciones sociales como la Universidad son apenas acuerdos temporales que no admiten racionalidades meramente estructurales, puesto que sus formaciones son inestables y cambiantes; por más que veamos una dinámica que se repite en la historia de la universidad, cuando esta se analiza en su devenir geneológico se 
comprende la gran movilidad que ha tenido en sus formas de decir, pero también en los nuevos objetos discursivos que va generando, en los tejidos continuos y discontinuos de la vida social.

Foucault (2008) también consideraría esta movilidad en sus estudios sobre la arqueología del saber, cuando muestra que una verdadera construcción discursiva se logra en las formas de ser de esa construcción; en las formas que ella va tomando como ruptura y continuidad; dinámica que lleva a que muchas formaciones se estabilicen con nuevas formas de decir o se trasformen definitivamente.

La Universidad como institución social entonces no es otra cosa que una configuración social (Elías, 1989, 1996), en cuanto acuerdo no permanente e inestable en el que se conjugan polifonías y múltiples dimensiones, todas ellas ancladas en una gran base imaginaria social.

Lo que vemos de la universidad actual siempre es resultado de las trasformaciones de esos imaginarios sociales en los cuales esa configuración está anclada y por tanto es perfectible y necesaria de resignificación. De esa universidad de papel referida por Porter (2005) o la servidumbre simbólica de la universidad denunciada por Baquero (2010), en reconocimiento del interés actual de estas publicaciones, es posible su reconfiguración en la lógica de entendimiento de su naturaleza no solamente simbólica sino fuertemente imaginaria. Pues es en esta dimensión donde se deben generar las trasformaciones para que estas prácticas que corresponden a una racionalidad meramente instrumental no se sigan presentando.

\section{Conclusiones}

Como se aprecia en el desarrollo del texto, la universidad como institución histórico-social ha trasegado por las razones más ortodoxas de la manipulación y el poder, inicialmente clerical y luego de los intereses políticos. Estos intentos por encerrar la universidad en la racionalidad de los organismos económico funcionales han buscado incluso dotarla de propiedades preestablecidas y estables, acopiando una serie de normatividades y postulados que solo se quedan en el papel.

La universidad por su naturaleza de institución social genera unos mecanismos de defensa a estos estatismos, los cuales se ubican en la base misma de las significaciones imaginarias sociales. Estos mecanismos son en realidad formas autodinámicas configuradas en el poder y convicción de las comunidades que la configuran y que le dan vida. Por eso, como lo muestran los estudios en referencia, los imaginarios instituidos de control y producción establecidos en los reglamentos solo son líneas de acción que los actores asumen como referentes caminando siempre por sus bordes, lo cual les permite reconfigurar a cada instante la universidad que las nuevas dinámicas sociales requieren.

Lo anterior es importante dadas las repercusiones que tiene para su organización y seguimiento, pues irse en contra de la naturaleza dinámica, que implica su relación con los imaginarios sociales, es desconocer que la universidad es una institución viva y en constante movimiento, el cual se percibe fácilmente en sus trasformaciones históricas. Basta con analizar la forma como sus funciones se han trasformado de acuerdo con lo que para las sociedades significa por ejemplo ciencia, conocimiento o saber. Las funciones 
asignadas por encima de estas relaciones siempre tienden a desvanecerse, al causar escozor en la comunidad.

\section{REFERENCIAS}

Arango, D. (2005). Aproximación histórica a la universidad colombiana. Los estudios superiores en el período colonial. Revista Historia de la Educación Latinoamericana, (7), 99. 136.

Baeza, M. (2000). Los caminos invisibles de la realidad social .Ensayo de sociología profunda sobre los imaginarios sociales. Chile: Ediciones sociedad de hoy.

Baquero, P. (2010). La servidumbre simbólica de la Universidad. Recuperado dehttp: //www.eldiplo.info/portal/index.php/ layout-1/layout-2/layout-4/layout-43/ regions-12345678/layout-47/root/ediciones-anteriores/item/341-la-servidumbre-simbólica-de-la-universidad.

Borrero, A. (2002). La universidad Latinoamericana, la reforma de Córdoba, legislación universitaria de México. Bogotá: Icfes.

Borrero, A. (2005). Modelos y valores universitarios en América Latina y Colombia durante los siglos XIX y XX. Simposio permanente sobre la Universidad. Pontificia Universidad Javeriana, Bogotá.

Castoriadis, C. (1983). La institución imaginaria de la sociedad. Marxismo y teoría revolucionaria (Vol. 1). Barcelona: TusQuets.

Castoriadis, C. (1989). La institución imaginaria de la sociedad. El imaginario social y la institución (Vol.2). Barcelona: TusQuets.

Castoriadis, C. (2008). El mundo fragmentado. La plata: Terramar.

Elias, N. (1989). El proceso de la civilización. Investigaciones sociogenéticas y psicogenéticas. México: Fondo de Cultura Económica.
Elias, N. (1996). La sociedad cortesana. Buenos Aires: Fondo de Cultura Económica.

Foucault, M. (2008). Arqueología del saber. Buenos aires: Siglo XXI.

Luzurriaga, L. (1946). Historia de la Educación pública. Buenos Aires: Editorial Losada S.A

Manchola, O. (1993). Reseña histórica de la Facultad de Bellas Artes. Revista Cincuentenario Universidad de Caldas. Recuperado de http://www.ucaldas.edu.co/ portal/historia-de-la-universidad/

Murcia-Peña, N., Murcia-Gómez, J. y Murcia-Gómez, N. (2009). Imaginarios sociales y autoevaluación universitaria. Educación y Educadores, 12(3), 99-115.

Murcia, P. (2012). Universidad y vida cotidiana. Imaginarios de profesores y estudiantes. España: EAE.

Murcia, P. (2006). Universidad y vida cotidiana. Estudio desde imaginarios de profesores $y$ estudiantes (Tesis doctoral). CIDE, Universidad de Manizales, Manizales.

Murcia, P., Sánchez, J. y Candamil, M. (2006). Autoevaluación en la universidad. Manizales: Universidad de Caldas.

Porter, L. (2005). La universidad de Papel. Centro de Investigaciones Interdisciplinarias en Ciencias y Humanidades de la Universidad Nacional Autónoma de México. México: Centro de Investigaciones Interdisciplinarias en Ciencias y Humanidades.

Romero, F. (2006). Reestructuración de la Universidad Latinoamericana: sus raíces y prospectiva. Recuperado de

http://www.ausenp.info/internas/admin/ informacion/uploader/REVISTA6.pdf

Shotter, J. (1993). Realidades conversacionales. La construcción de la vida a través del lenguaje. Buenos Aires: Amorrortu.

Zarruk, G. P. (1992). Marco Ideológico-político del surgimiento de la universidad. 
Teoría sobre el origen del poder en la edad media. En Asociación Colombiana de Universidades Ascun. Simposio permanente sobre Universidad. Quinto seminario general 1990-1992. Fundación para la educación superior FES, Instituto Colombiano para el Fomento de la Educación Superior [ICFES], Bogotá. 